\title{
GALAXIES
}

\section{Ultra-diffuse and hyper-mysterious}

Mon. Not. R. Astron. Soc. (in the press); preprints available at

https://arxiv.org/abs/1906.11543 and https://arxiv.org/abs/1907.01559

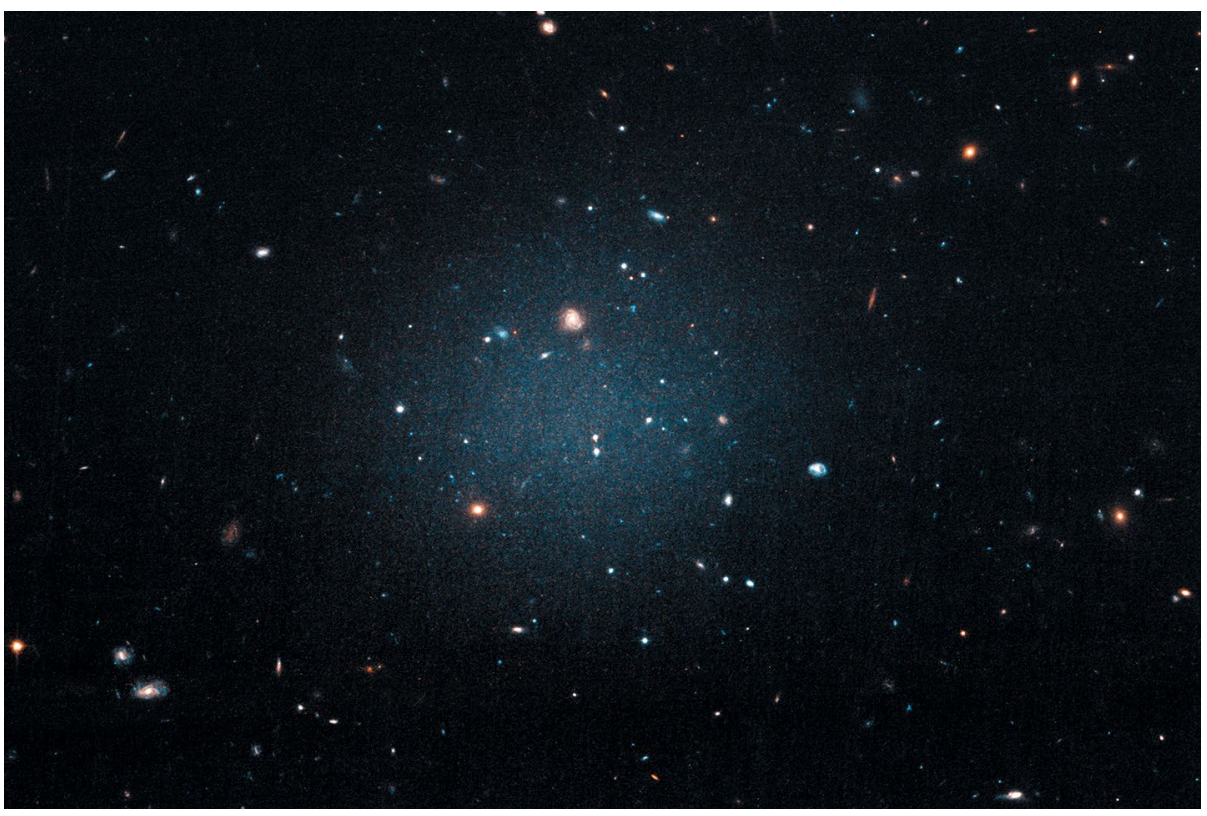

Credit: NASA, ESA, and P. van Dokkum (Yale University)

Ultra-diffuse galaxies (UDGs; pictured) are extremely low-luminosity and low-surfacebrightness galaxies that have been known to exist at least since 1984, when Allan Sandage and Bruno Binggeli discovered one such system in the Virgo cluster. However, the formation and evolution of these galaxies is still a topic of debate and might be key in our understanding of the evolution of galaxies in general. Two independent papers, led by Steven Janowiecki and Daniel Prole, report that the environment in which UDGs are born appears to be of little importance.

Prole et al. use optical imaging data from the Kilo-Degree Survey to calculate the abundance and properties of UDGs in the field (away from clusters or groups of galaxies). They find that isolated UDGs are predominantly blue and star-forming. By comparison with UDGs and normal galaxies in clusters, the authors conclude that UDGs are more efficiently quenched in clustered environments than their more luminous counterparts. Interestingly, the efficiency with which UDGs are formed in the field is found to be the same as for those in clusters.

In an independent study, Janowiecki et al. look at the environments of UDGs selected using $\mathrm{H}$ I atomic gas emission, based on spectroscopic data from the ALFALFA survey. The selected UDGs span different types of environments and indeed the authors conclude that the environments that UDGs inhabit are statistically the same as other samples of $\mathrm{H}$ I-selected galaxies. The authors conclude that the properties of $\mathrm{H}$ I-selected UDGs are mainly driven by internal processes. While gas-poor UDGs in clusters may be a result of environmental processes, the initial formation of UDGs does not seem to require any specific environment.

Marios Karouzos

Published online: 26 July 2019

https://doi.org/10.1038/s41550-019-0872-2 